\title{
INFLUÊNCIA DE ALGUNS PARÂMETROS EXPERIMENTAIS NOS RESULTADOS DE ANÁLISES CALORIMÉTRICAS DIFERENCIAIS - DSC
}

Cláudia Bernal, Andréa Boldarini Couto, Susete Trazzi Breviglieri e Éder Tadeu Gomes Cavalheiro*

Departamento de Química, Universidade Federal de São Carlos, CP 676, 13565-905 São Carlos - SP

Recebido em 18/10/01; aceito em 3/1/02

INFLUENCE OF SOME EXPERIMENTAL PARAMETERS ON THE RESULTS OF DIFFERENTIAL SCANNING CALORIMETRY - DSC. A series of experiments were performed in order to demonstrate to undergraduate students or users of the differential scanning calorimetry (DSC), that several factors can influence the qualitative and quantitative aspects of DSC results. Saccharin, an artificial sweetner, was used as a probe and its thermal behavior is also discussed on the basis of thermogravimetric (TG) and DSC curves.

Keywords: saccharin; differential scanning calorimetry; results evaluation.

\section{INTRODUÇÃO}

Há algum tempo foi apresentado um artigo sobre a influência de parâmetros experimentais, em resultados termogravimétricos ${ }^{1}$. Naquela oportunidade procurou-se demonstrar como parâmetros controláveis pelo operador podem modificar os resultados obtidos em termogravimetria, bem como foi apresentada uma classificação das diversas técnicas que compreendem o conjunto de técnicas termoanalíticas. A análise térmica, foi definida por Mackenzie ${ }^{2}$ como sendo "um grupo de técnicas nas quais uma propriedade física de uma substância elou seus produtos de reação é medida, enquanto a amostra é submetida a uma programação de temperatura".

Dentre as técnicas termoanalíticas mais utilizadas encontram-se a Análise Térmica Diferencial (DTA - do inglês "Differential Thermal Analysis"), na qual se acompanha a variação de temperatura da amostra em relação a um material inerte de referência, e a Calorimetria Exploratória Diferencial (DSC - do inglês "Differential Scanning Calorimetry"), na qual se acompanha a variação da energia entre a amostra e a referência. A nomenclatura e as abreviaturas seguem o padrão sugerido por Ionashiro \& Giolito ${ }^{3}$, para a língua portuguesa, segundo recomendação da Associação Brasileira de Análise Térmica e Calorimetria - ABRATEC".

Historicamente, as curvas de aquecimento de Le Chatelier acompanhavam a variação na temperatura de uma amostra em função do tempo, enquanto a mesma era aquecida. Caso não ocorra nenhum fenômeno físico ou químico com a amostra observa-se uma reta para a variação de temperatura em relação ao tempo, como na Figura 1a. Havendo liberação de calor, processo exotérmico, verificava-se um aumento na temperatura durante o processo, representada por uma inflexão no perfil temperatura - tempo, como representado na Figura 1b.

Para um processo endotérmico, com absorção de calor, observase a diminuição na temperatura da amostra e a inflexão na curva pode ser representada pela Figura 1d.

Na Análise Térmica Diferencial o que se acompanha é a variação na propriedade física temperatura da amostra, em relação a um material que não apresenta absorção ou liberação de calor (termicamente inerte), a referência ${ }^{4}$.

\footnotetext{
*e-mail: cavalheiro@dq.ufscar.br
}

\# sítio internet: www.abratec.com.br
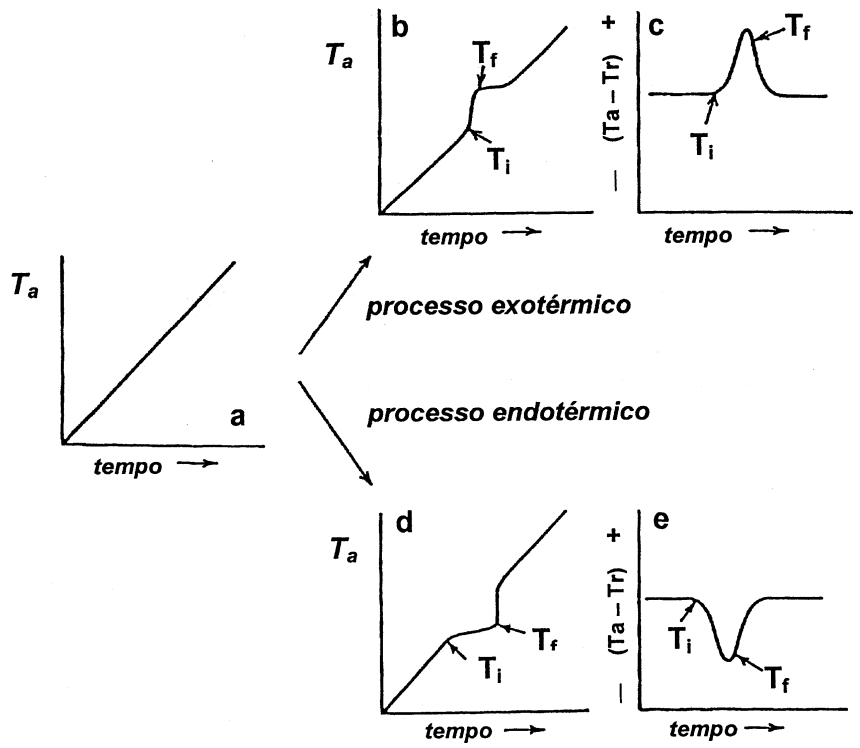

Figura 1. Curva de aquecimento: a) quando não ocorre nenhum evento térmico; b) quando ocorre processo exotérmico; c) idem b, porém para sistema térmico diferencial; d) quando ocorre processo endotérmico; e) idem $d$, porém para sistema térmico diferencial

A diferença de temperatura $(\Delta \mathrm{T})$, é dada pela temperatura da amostra $\left(\mathrm{T}_{\mathrm{a}}\right)$ menos a temperatura da referência $\left(\mathrm{T}_{\mathrm{r}}\right)$ :

$\Delta \mathrm{T}=\mathrm{T}_{\mathrm{a}}-\mathrm{T}_{\mathrm{r}}$

caso não haja ocorrência de fenômeno físico ou químico, observa-se uma reta paralela ao eixo do tempo. Um processo exotérmico é representado por um pico para cima, Figura 1c; enquanto um processo endotérmico é representado por um pico para baixo, Figura 1e.

Em um experimento de Calorimetria Exploratória Diferencial, mede-se a variação de entalpia que ocorre entre a amostra e a referência durante o processo de aquecimento/resfriamento. Diferentes arranjos de construção utilizam o mesmo nome para o equipamento. O termo DSC foi utilizado primeiramente em 1963, para definir um sistema desenvolvido pela empresa norte-americana Perkin-Elmer. Atualmente tem-se definido ${ }^{4,5}$ : 
DSC com Compensação de Potência: arranjo no qual a referência e amostra são mantidas na mesma temperatura, através de aquecedores elétricos individuais. A potência dissipada pelos aquecedores é relacionada com a energia envolvida no processo endotérmico ou exotérmico.

DSC com Fluxo de Calor: o arranjo mais simples é aquele no qual a amostra e a referência, contidas em seus respectivos suportes de amostra, são colocadas sobre um disco de metal. A troca de calor entre o forno e a amostra ocorre preferencialmente pelo disco. Para uma condição de fluxo de calor de estado estacionário, o sinal medido $\Delta T$ (diferença de temperatura entre a amostra e a referência), é proporcional à diferença nos fluxos de calor, $\Delta \phi$, entre a amostra, $\phi_{A}$, e referência, $\phi_{R}$, e é representada pela Equação 2:

$\Delta \phi=\phi_{A}-\phi_{R}=-k \Delta T$

Um processo endotérmico na amostra, cria um $\Delta T<0$ e $\Delta \phi>0$, ou seja flui calor do disco para a amostra. O recíproco ocorre para um processo exotérmico. A constante $\mathrm{k}$ deve ser determinada por calibração, usando-se padrões com uma constante física conhecida, por exemplo entalpia de fusão.

Uma discussão detalhada sobre os princípios do DSC com compensação de potência e para outros arranjos de DSC com fluxo de calor é apresentada por Hemminger 5 .

A Figura 2 apresenta um exemplo de equipamento genérico para análise térmica diferencial e sua classificação de acordo com a célula utilizada nas medidas.

As curvas DSC apresentam forma semelhante às descritas para DTA. Entretanto quando ocorre um processo de absorção de calor, endotérmico, surge um pico positivo (já que o aquecedor da amostra deve dissipar calor para manter a temperatura igual à referência), enquanto no processo de emanação de calor, exotérmico, o pico é negativo. Isto torna necessário marcar o sentido dos processos no gráfico resultante ${ }^{3}$. As unidades dos eixos das ordenadas são:

$d Q / d t$ em função do tempo/Temperatura para DSC;

$\Delta T=T_{a}-T_{r}$, em função do tempo/Temperatura para DTA.
O DSC permite determinações quantitativas, estando a área dos picos relacionada com a energia envolvida no processo, sendo utilizados padrões para calibração do equipamento.

Estes padrões apresentam variação de entalpia conhecida, normalmente de fusão, e a área do pico deste processo é comparada com a área do processo apresentado pela amostra. A Tabela 1 apresenta alguns exemplos de padrões normalmente utilizados.

Tabela 1. Padrões normalmente usados em calibração de temperatura e energia e as constantes físicas relacionadas com estes parâmetros ${ }^{8}$

\begin{tabular}{lcc}
\hline Padrão & Ponto de Fusão $/{ }^{\circ} \mathrm{C}$ & $\Delta \mathrm{H}_{\text {fus }} / \mathrm{J} \mathrm{g}^{-1^{*}}$ \\
\hline Índio & 156,4 & 28,50 \\
Estanho & 231,9 & 60,25 \\
Chumbo & 327,4 & 22,80 \\
Zinco & 419,5 & 108,40 \\
Alumínio & 660,4 & 397,00 \\
\hline
\end{tabular}

* algarismos significativos como na referência original

Várias teorias têm sido apresentadas relacionando as áreas dos picos com as energias envolvidas nos processos fisico-químicos medidos em DSC, devendo-se considerar que a forma da curva é função de parâmetros da amostra e do equipamento, tal como capacidade calorífica, etc..

Dentre estas propostas, uma das mais simples foi apresentada por Speil et al. ${ }^{6}$ e Kerr \& Kulp ${ }^{7}$, que relacionaram a variação de entalpia envolvida com a área do pico por:

$\frac{\mathrm{m}(\Delta \mathrm{H})}{\mathrm{gk}}=\int_{\mathrm{T}_{1}}^{\mathrm{T}_{2}} \Delta \mathrm{TdT}$

na qual $\Delta \mathrm{H}$ é a variação de entalpia, m é a massa da amostra, $\Delta \mathrm{T}$ é a variação de temperatura entre o início $\left(\mathrm{T}_{1}\right)$ e o final $\left(\mathrm{T}_{2}\right)$ do processo, g e k são constantes referentes à amostra, ao porta-amostra e ao instrumento utilizado. Outras relações mais complexas são apresentadas e discutidos por Wendlandt ${ }^{4}$, Brown $^{8}$ e Haines 9

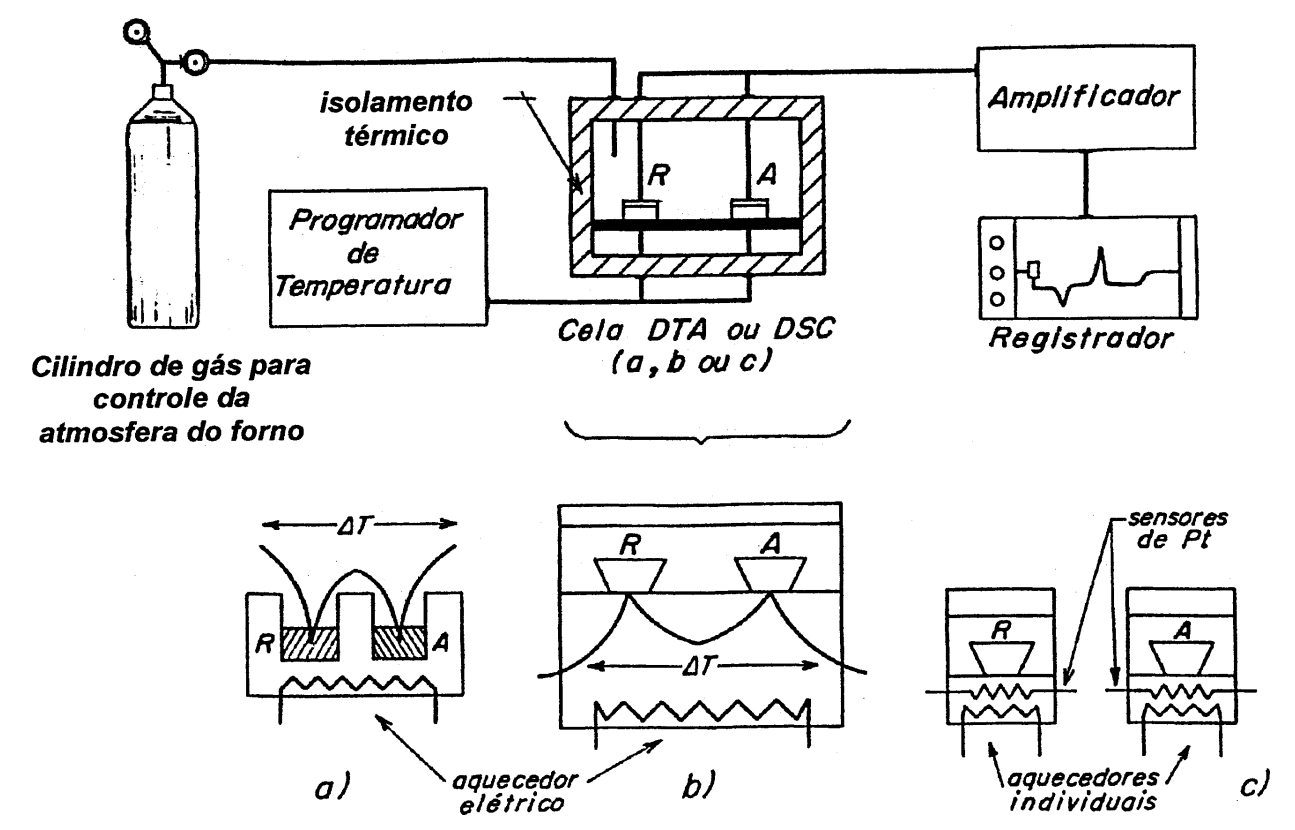

Figura 2. Esquema de um equipamento genérico para análise térmica diferencial (DTA) e calorimetria exploratória diferencial (DSC). a) DTA; b) DSC com fluxo de calor; c) DSC com compensação de potência 
Uma curva típica resultante de um experimento DSC para uma amostra genérica é representada na Figura 3. A forma desta curva pode ser afetada por fatores instrumentais e por características da amostra, assim como ocorre para as curvas $\mathrm{TG}^{1}$, uma vez que se tratam de técnicas de temperatura dinâmica.

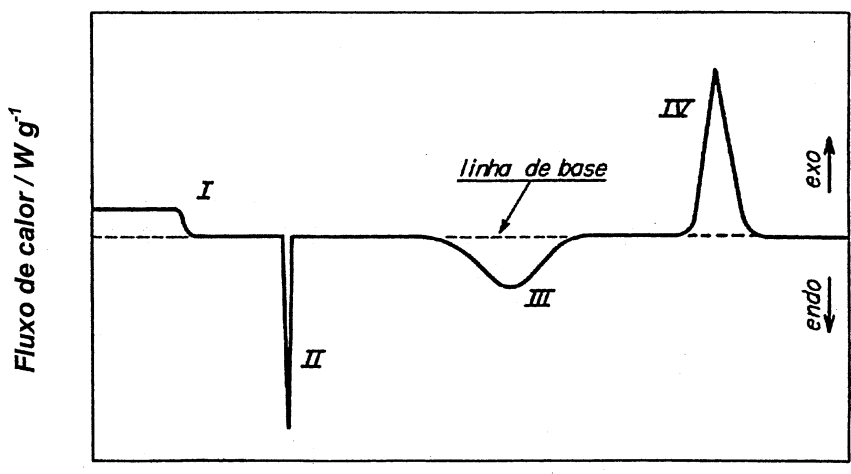

tempo

Figura 3. Curva genérica para um experimento DSC/DTA. I) mudança de linha de base sem pico; II e III) picos endotérmicos; IV) pico exotérmico

Nestes experimentos a forma, posição e número de picos são úteis para análise qualitativa, enquanto a área sob os mesmos é de interesse quantitativo no caso do DSC e, portanto, torna-se importante conhecer como fatores externos podem afetar estas características para análise adequada dos resultados e sua reprodutibilidade.

Um resumo dos principais eventos térmicos observados em DTA e sua forma característica foi apresentado por Gordon ${ }^{10}$, que os classificou de acordo com sua origem física ou química e sua natureza exotérmica ou endotérmica. Tal classificação é reproduzida na Tabela 2.

Tabela 2. Caráter exotérmico ou endotérmico dos picos DTA (ou DSC), de acordo com sua origem física ou química, segundo Gordon $^{10}$

\begin{tabular}{|c|c|c|}
\hline \multirow[t]{2}{*}{ Fenômeno } & \multicolumn{2}{|c|}{ Variação de entalpia } \\
\hline & endotérmica & exotérmica \\
\hline \multicolumn{3}{|l|}{ Físico } \\
\hline $\begin{array}{l}\text { transição cristalina } \\
\text { fusão } \\
\text { vaporização } \\
\text { sublimação } \\
\text { adsorção } \\
\text { dessorção } \\
\text { absorção } \\
\text { transição de Ponto Curie } \\
\text { transição vítrea } \\
\text { transição de cristal líquido }\end{array}$ & $\begin{array}{c}\mathrm{X} \\
\mathrm{X} \\
\mathrm{X} \\
\mathrm{X} \\
\mathrm{X} \\
\mathrm{X} \\
\mathrm{X} \\
\text { mudança de li } \\
\mathrm{X}\end{array}$ & $\begin{array}{c}\mathrm{X} \\
\mathrm{X} \\
\mathrm{e}, \text { sem picos }\end{array}$ \\
\hline \multicolumn{3}{|l|}{ Químico } \\
\hline $\begin{array}{l}\text { quimissorção } \\
\text { dessolvatação } \\
\text { desidratação } \\
\text { decomposição } \\
\text { degradação oxidativa } \\
\text { oxidação em atmosfera gasosa } \\
\text { redução em atmosfera gasosa } \\
\text { reação de óxido-redução } \\
\text { combustão } \\
\text { polimerização } \\
\text { pré-cura (resinas) } \\
\text { reações catalíticas }\end{array}$ & $\begin{array}{l}X \\
X \\
X\end{array}$ & $\begin{array}{l}X \\
X \\
X \\
X \\
X \\
X \\
X \\
X \\
X\end{array}$ \\
\hline
\end{tabular}

Neste caso, assim como na discussão de $\mathrm{TG}^{1}$, os livros texto de graduação, normalmente adotados, apresentam discussões superficiais sobre o tema ${ }^{11,12}$, enquanto livros específicos de análise térmica nem sempre apresentam curvas ilustrando esta influência ${ }^{8,9}$. Wendlandt ${ }^{4}$ é um autor que se preocupa em apresentar e ilustrar esta discussão, porém seu livro não está disponível em todas as bibliotecas universitárias.

Desta forma, o presente trabalho visa demonstrar, com experimentos simples, como alguns fatores experimentais controláveis pelo operador, podem alterar os resultados DSC e a importância de fixar condições experimentais para reprodutibilidade dos experimentos térmicos diferenciais. Os pontos aqui apresentados podem ser estendidos para experimentos DTA.

Algumas das principais aplicações das técnicas termoanalíticas diferenciais DTA e DSC estão resumidas na Tabela 3.

Tabela 3. Principais aplicações das técnicas termoanalíticas diferenciais ${ }^{4,8}$

\begin{tabular}{ll}
\hline Aplicação & Fenômeno investigado \\
\hline catalisadores & reações de decomposição \\
polímeros & processos fisico-químicos \\
graxas e lubrificantes & processos físição-químicos \\
óleos e gorduras & reações de desidratação e decom- \\
composição & danos causados por radiação \\
carboidratos & catálise \\
aminoácidos e proteínas & desidratação \\
sais hidratados & calores de reação \\
óxidos & calores de reação \\
carvão e petróleo & calores de transição \\
produtos naturais & estabilidade térmica e oxidativa, \\
materiais biológicos e fármacos & comparação, determinação de \\
& pureza \\
\hline
\end{tabular}

A crescente utilização das técnicas termoanalíticas justifica plenamente tal discussão, pois alunos de graduação nem sempre têm acesso às técnicas, encontrando dificuldades no trabalho como profissionais. Devido à sua importância, questões envolvendo as técnicas termoanalíticas estão incluídas no programa do Exame Nacional de Cursos, o "Provão", referente aos cursos de graduação em química.

Além dos fatores aqui discutidos, há os fatores instrumentais que normalmente não dependem do operador, tais como geometria de forno e porta-amostra, condutividade térmica do forno, características de termopares, capacidades caloríficas de materiais usados na construção dos equipamentos, etc...

\section{Considerações sobre o composto usado como exemplo - a sacarina}

Nos experimentos demonstrativos aqui apresentados foi empregada a sacarina, um adoçante artificial muito comum em produtos dietéticos, cuja estrutura é apresentada na Figura 4. Alguns aspectos históricos de sua utilização são descritos por Tarbell e Tarbell ${ }^{13} \mathrm{e}$ Snyder ${ }^{14}$.<smiles>O=C1NS(=O)(=O)c2ccccc21</smiles>

Figura 4. Fórmula estrutural da sacarina 
Segundo estes autores a sacarina foi sintetizada pela primeira vez em 1879, acidentalmente, por Ira Remsen e Constantine Fahlberg. Logo após a sua descoberta, a sacarina foi utilizada para conservação de alimentos e, então, como um substituto do açúcar, uma vez que a sua doçura é cerca de 500 vezes superior.

Na década de 70, o uso da sacarina foi bastante difundido, principalmente em alimentos de baixas calorias e dietéticos, enquanto surgiam dúvidas sobre a segurança do seu uso. Testes biológicos levaram à conclusão de que altas doses, em uso contínuo, induziram câncer em cobaias de laboratório. Entretanto, estudos posteriores comprovaram que o uso da sacarina apresenta um risco considerado aceitável e a sacarina foi considerada segura, quando usada em doses adequadas.

\section{PARTE EXPERIMENTAL}

As curvas TG/DTG foram obtidas em um módulo termogravimétrico TGA-951 (TA - Instruments), acoplado a um analisador térmico TA2000 (TA - Instruments), utilizando-se razão de aquecimento de $10{ }^{\circ} \mathrm{C} \mathrm{min}^{-1}$, sob atmosfera dinâmica de ar e de nitrogênio, com vazão de gás da ordem de $100 \mathrm{ml} \mathrm{min}{ }^{-1}$, sob pressão atmosférica. As massas de amostra foram da ordem de $7 \mathrm{mg}$ com precisão de \pm 1 $\mu$ g. A temperatura máxima foi de $450{ }^{\circ} \mathrm{C}$, em suporte de amostra de platina.

Com o objetivo de elucidar o mecanismo de decomposição, uma amostra de aproximadamente $0,5 \mathrm{~g}$ de sacarina foi aquecida em tubo de ensaio $(20 \times 2 \mathrm{~cm})$, imerso em glicerina, com um termômetro acoplado ao tubo.

As curvas DSC foram obtidas em um módulo calorimétrico exploratório diferencial DSC 910 (TA - Instruments) (tipo fluxo de calor), acoplado a um analisador térmico TA2000 (TA - Instruments). Nos ensaios, utilizou-se sacarina da Aldrich com pureza de no mínimo $99 \%$, suporte de amostra de alumínio, atmosfera de ar e nitrogênio. O equipamento foi previamente calibrado para temperatura utilizando como padrões os pontos de fusão de índio $\left(156,4^{\circ} \mathrm{C}\right)$ e zinco $(419,5$ ${ }^{\circ} \mathrm{C}$ ) metálicos, com pureza de $99,99 \%$. A calibração para energia foi feita com base na entalpia de fusão do índio metálico ${ }^{8}, \Delta \mathrm{H}_{\text {fus }}=28,5 \mathrm{~J}$ $\mathrm{g}^{-1}$. Foram calculados os fatores de correção conforme procedimento e especificação do fabricante. Para demonstração da influência dos diferentes parâmetros, em cada caso, fixou-se uma série de parâmetros variando-se o fator analisado, como descrito para cada um a seguir.

\section{RESULTADOS E DISCUSSÃO}

\section{Comportamento termogravimétrico da sacarina}

A Figura 5a apresenta as curvas TG/DTG sob atmosfera de nitrogênio, sugerindo uma única perda de massa entre $163-280{ }^{\circ} \mathrm{C}$, com resíduo de $1,7 \%$ ao final do processo.

A Figura $5 b$ apresenta as curvas TG/DTG sob atmosfera de ar sintético também com uma única perda de massa entre $163-288^{\circ} \mathrm{C}$, com resíduo de $0,64 \%$.

Durante o aquecimento em tubo de ensaio com a amostra de sacarina, verificou-se a fusão do material seguida de volatilização com condensação nas paredes do tubo de ensaio. $\mathrm{O}$ condensado foi submetido a análise por espectroscopia na região do infravermelho (IR), análise elementar (AE) e ressonância magnética nuclear de prótons $\left(\mathrm{RMN}-{ }^{1} \mathrm{H}\right)$. Os resultados comprovam que ocorreu fusão seguida de volatilização. Todas estas técnicas apresentaram resultados idênticos para o condensado e o material de partida, confirmando a presença da sacarina no tubo. A análise por teste de chama do resíduo de $\mathrm{TG}$ a $280{ }^{\circ} \mathrm{C}$, mostrou a presença de íons sódio, provavelmente contidos na amostra como sacarinato de sódio.

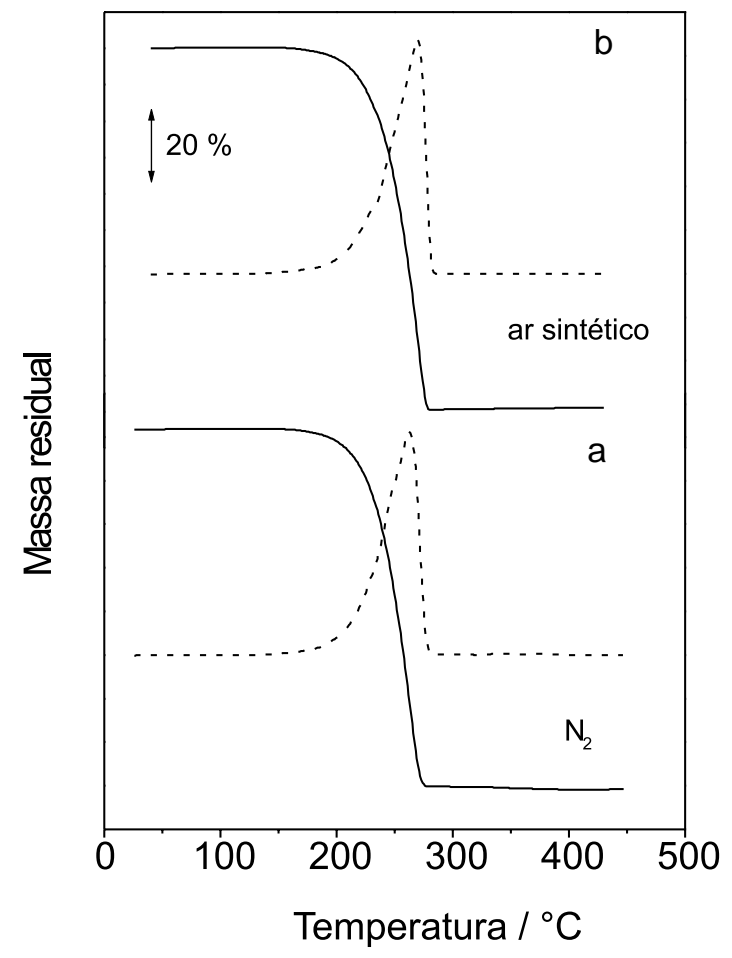

Figura 5. Curvas TG (linha cheia) e DTG (linha tracejada) da sacarina sob atmosfera dinâmica de ar sintético e $N_{2}$. Massa de amostra $7 \mathrm{mg}$, vazão de gás $100 \mathrm{~mL} \mathrm{~min}^{-1}$, suporte de amostra de platina, razão de aquecimento 10 ${ }^{\circ} \mathrm{C} \mathrm{min}^{-1}$

O comportamento térmico da sacarina poderia ser resumido pelas equações químicas abaixo:

$$
\begin{aligned}
& \mathrm{C}_{7} \mathrm{H}_{5} \mathrm{SNO}_{3(\mathrm{~s})} \rightarrow \mathrm{C}_{7} \mathrm{H}_{5} \mathrm{SNO}_{3(\mathrm{l})} \\
& \mathrm{C}_{7} \mathrm{H}_{5} \mathrm{SNO}_{3(\mathrm{l})} \rightarrow \mathrm{C}_{7} \mathrm{H}_{5} \mathrm{SNO}_{3(\mathrm{~g})}+\text { resíduo } \\
& \mathrm{C}_{7} \mathrm{H}_{5} \mathrm{SNO}_{3(\mathrm{~g})} \rightarrow \mathrm{C}_{7} \mathrm{H}_{5} \mathrm{SNO}_{3(\mathrm{~s})}
\end{aligned}
$$

O composto com pureza de $99 \%$ foi escolhido propositadamente, já que as impurezas presentes ficam como resíduo após volatilização da amostra e se decompõem de maneira diferente em atmosfera de ar ou nitrogênio, como apresentado a seguir.

\section{Variação da razão de aquecimento no DSC}

Foi utilizada massa de sacarina de 3,0 mg, aquecida sob atmosfera dinâmica de ar sintético $\left(100 \mathrm{~mL} \mathrm{~min}^{-1}\right)$, variando a razão de aquecimento de 2,$5 ; 5,0 ; 10 ; 15 ; 20$ e $40{ }^{\circ} \mathrm{C}$ min $^{-1}$ em cada experimento. Os resultados são apresentados na Figura 6.

As curvas DSC obtidas com diferentes razões de aquecimento apresentaram dois picos endotérmicos. $\mathrm{O}$ primeiro relacionado com a fusão e o segundo atribuído à volatilização, de acordo com os resultados de IR, RMN- ${ }^{1} \mathrm{H}$ e AE e um último pico exotérmico correspondente à decomposição de material residual referente às impurezas, pois a sacarina já haveria volatilizado nesta temperatura. Podese observar que, de maneira geral, não houve deslocamento na temperatura de fusão (primeiro pico) com o aumento da razão de aquecimento. Nota-se que quanto menor for a razão de aquecimento mais agudo e menos intenso o pico de fusão. Altas razões de aquecimento alargam a forma do pico ${ }^{4}$. 


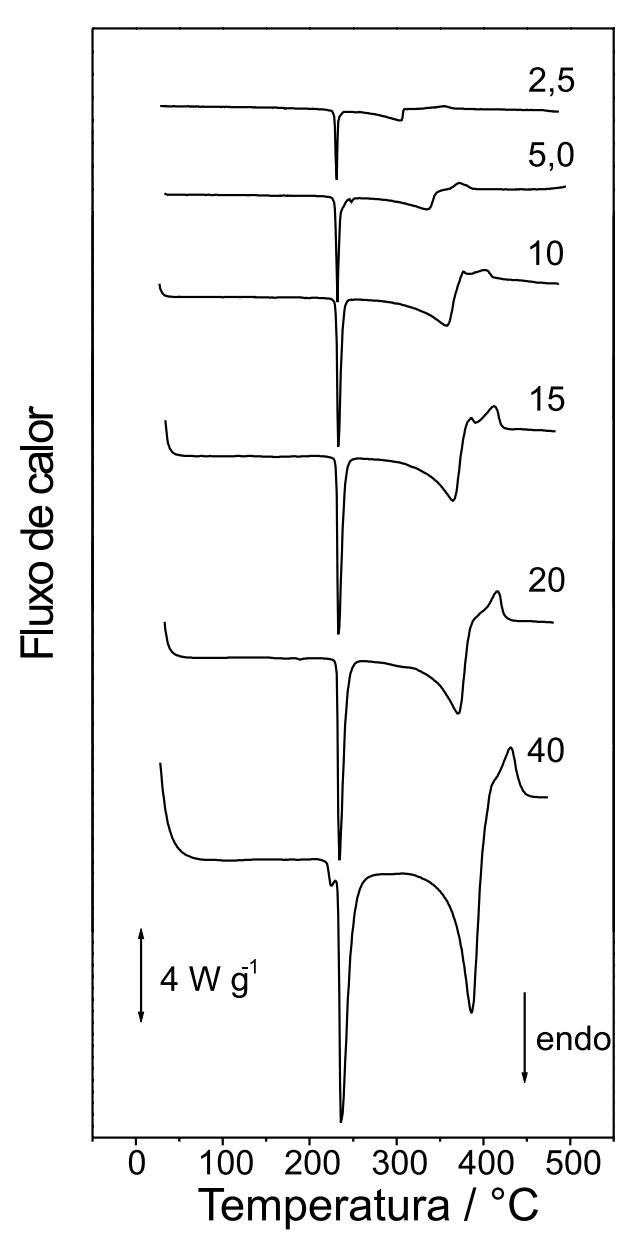

Figura 6. Curvas DSC da sacarina em diferentes razões de aquecimento $\left(2,5-40{ }^{\circ} \mathrm{C} \mathrm{min}^{-1}\right)$. Massa de amostra $3 \mathrm{mg}$, suporte de amostra de alumínio com tampa, atmosfera dinâmica de ar sintético $\left(100 \mathrm{~mL} \mathrm{~min}{ }^{-1}\right)$

O segundo evento é afetado com o aumento da razão de aquecimento. Quanto maior a razão de aquecimento utilizada, maior a temperatura em que aparece a transição. O mesmo é observado para o processo exotérmico referente à queima do resíduo.

Devido à intensificação na magnitude dos picos pode-se observar, Figura 6, o surgimento de processos não detectáveis em razões de aquecimento baixas. Sob razões de aquecimento elevadas pode ocorrer mascaramento de picos, por sobreposição. Portanto, é imprescindível definir experimentalmente a razão de aquecimento adequada a cada amostra, para se observar o máximo de eventos térmicos.

Com relação ao aspecto quantitativo segundo Speil et al. ${ }^{6}$ a temperatura de pico representa o ponto no qual a entrada de calor é igual à razão de absorção de calor. Rearranjando a Equação 3, tem-se:

$\Delta T_{\min }=\left(\frac{d H}{d t}\right)_{\max } \frac{m}{g k}$

Uma maior razão de aquecimento provoca um aumento na relação $\mathrm{dH} / \mathrm{dt}$, pois mais reação ocorre no mesmo intervalo de tempo e, portanto, o ápice de temperatura será maior. Uma vez que o retorno à linha base é função do tempo assim como da diferença de temperatura, o retorno ocorre em temperatura real maior em aquecimentos mais rápidos e a área dos picos deve se manter próxima, apesar de aparentemente serem menores em razões de aquecimento menores. Para diferentes razões de aquecimento, a área para o processo refe- rente à desidratação da caulinita apresentou um desvio de $\pm 3 \%$ entre 5 e $20{ }^{\circ} \mathrm{C} \mathrm{min}^{-1}$, em experimentos de DTA. ${ }^{6}$

No presente caso os processos de fusão e volatilização, que se sobrepõem, não permitem demonstrar claramente este aspecto.

\section{Variação de massa de amostra no DSC}

Nestes experimentos utilizou-se razão de aquecimento constante de $10{ }^{\circ} \mathrm{C} \mathrm{min}{ }^{-1}$ e atmosfera dinâmica de ar sintético, com vazão de $100 \mathrm{~mL} \mathrm{~min}^{-1}$. As massas de amostra empregadas foram 3,0; 7,0 e $10 \mathrm{mg}$.

Os resultados obtidos (Figura 7) mostram que, quanto menor a massa da amostra, menor a temperatura em que a transição referente à decomposição ocorre, o que resulta da maior facilidade da homogeneização de temperatura no material, quando a amostra é de menor massa.

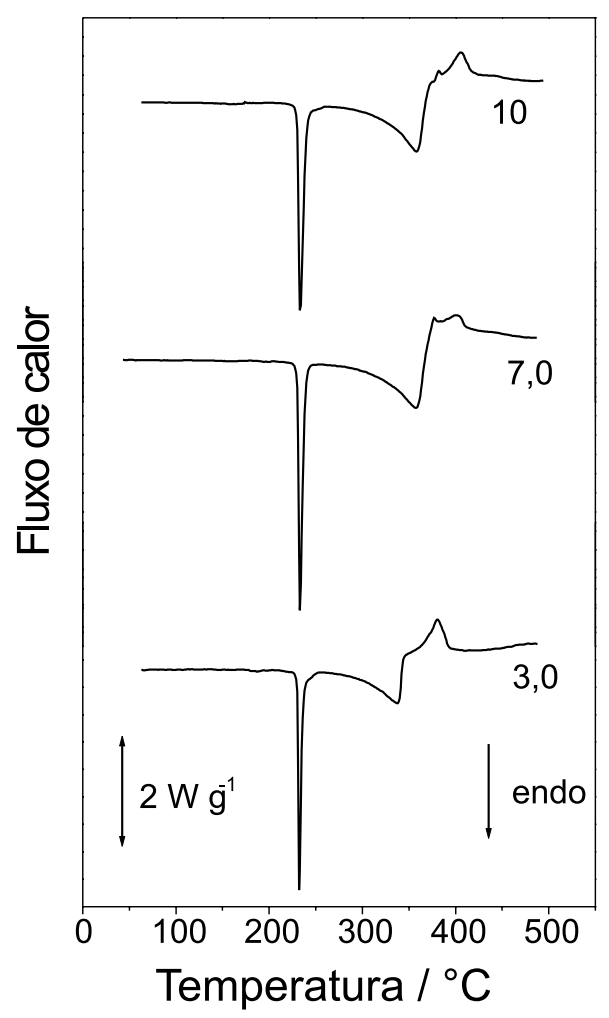

Figura 7. Curvas DSC da sacarina com diferentes massas de amostra (3 $10 \mathrm{mg}$ ). Suporte de amostra de alumínio com tampa, atmosfera dinâmica de ar sintético $\left(100 \mathrm{~mL} \mathrm{~min}{ }^{-1}\right)$, razão de aquecimento $10^{\circ} \mathrm{C} \mathrm{min}^{-1}$

\section{Variação da vazão do gás de controle da atmosfera do forno no DSC}

Para estes experimentos foi utilizada massa de amostra de 3,0 $\mathrm{mg}$, com razão de aquecimento de $10{ }^{\circ} \mathrm{C} \mathrm{min}^{-1}$ e atmosfera dinâmica de ar sintético com vazão variando entre 50; 100 e $150 \mathrm{ml} \mathrm{min}^{-1}$. Para verificar melhor a influência da vazão, utilizou-se suporte de amostra aberto. Os resultados podem ser vistos na Figura 8.

Pode-se notar que o processo de volatilização se completa em temperaturas ligeiramente menores, quando se usa uma vazão de gás mais alta, com o retorno à linha base também ocorrendo mais rapidamente. Isto pode ser explicado por uma maior eficiência na remoção da sacarina volatilizada. 


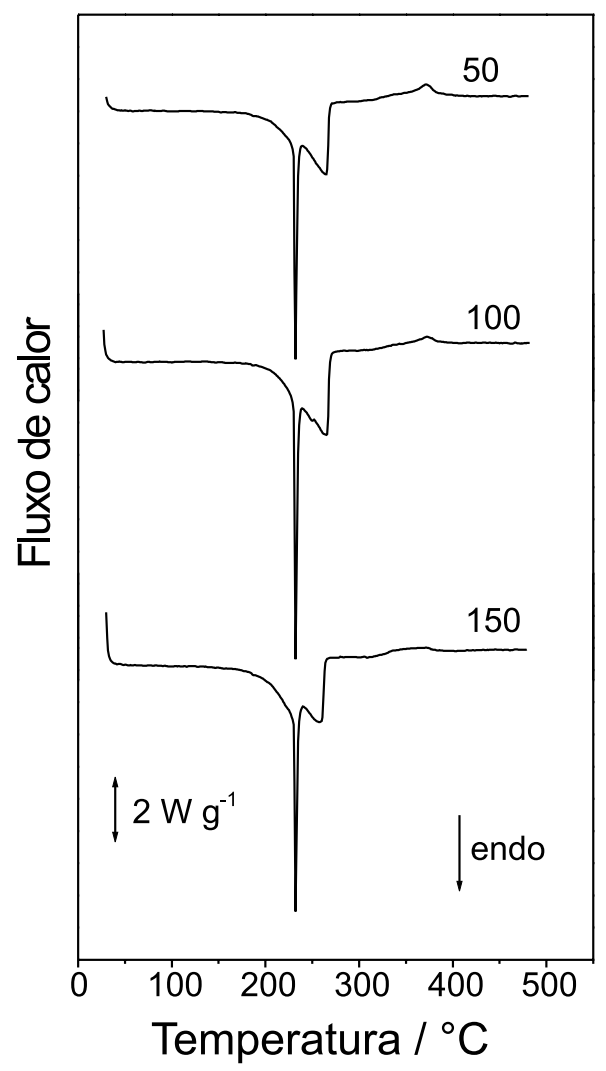

Figura 8. Curvas DSC da sacarina sob diferentes vazões de ar sintético (50 - $150 \mathrm{~mL} \mathrm{~min} \mathrm{~m}^{-1}$ ). Massa de amostra $3 \mathrm{mg}$, suporte de amostra de alumínio aberto, razão de aquecimento $10^{\circ} \mathrm{C} \mathrm{min}{ }^{-1}$

\section{Variação no adensamento da amostra no DSC}

Nestas medidas utilizou-se uma massa de amostra fixa de 3,0 mg, razão de aquecimento de $10{ }^{\circ} \mathrm{C} \mathrm{min}{ }^{-1} \mathrm{e}$ atmosfera dinâmica de ar com vazão de $100 \mathrm{~mL} \mathrm{~min}^{-1}$. Uma das amostras foi macerada até a obtenção de sacarina finamente dividida e a outra prensada na forma de pastilha, em um pastilhador de infravermelho.

Na Figura 9 não se observam mudanças significativas na forma dos picos de fusão e sublimação. Entretanto a queima do material residual (acima de $350{ }^{\circ} \mathrm{C}$ ) é afetada. Surgem dois picos exotérmicos a 364 e $389{ }^{\circ} \mathrm{C}$ com um vale endotérmico entre eles. Este fato foi atribuído a uma blindagem do material prensado no interior da pastilha, o qual sublima e carboniza após a queima da fração mais externa da amostra. A fusão e sublimação do material mais interno seria representada pelo vale endotérmico em torno de $380^{\circ} \mathrm{C}$. No material granulado o processo de queima ocorre em uma só etapa a $372{ }^{\circ} \mathrm{C}$.

\section{Influência dos gases da atmosfera do forno no DSC}

Neste caso utilizaram amostras com 3,0 mg, razão de aquecimento de $10{ }^{\circ} \mathrm{C} \mathrm{min}{ }^{-1}$ com vazão de gás de arraste de $100 \mathrm{~mL} \mathrm{~min}{ }^{-1}$. Os gases utilizados foram nitrogênio e ar sintético.

A influência da atmosfera do forno pode ser observada na Figura 10 .

Este parâmetro exerce um papel importante, podendo levar a modificações do perfil da curva DSC, em atmosfera de ar, devido a processos exotérmicos de degradação oxidativa de compostos orgânicos, combustão, oxidação de cátions metálicos, entre outros, enquanto que em atmosfera inerte como de nitrogênio estes processos não ocorrem. Isto pode ser observado na Figura 10, a qual apresenta

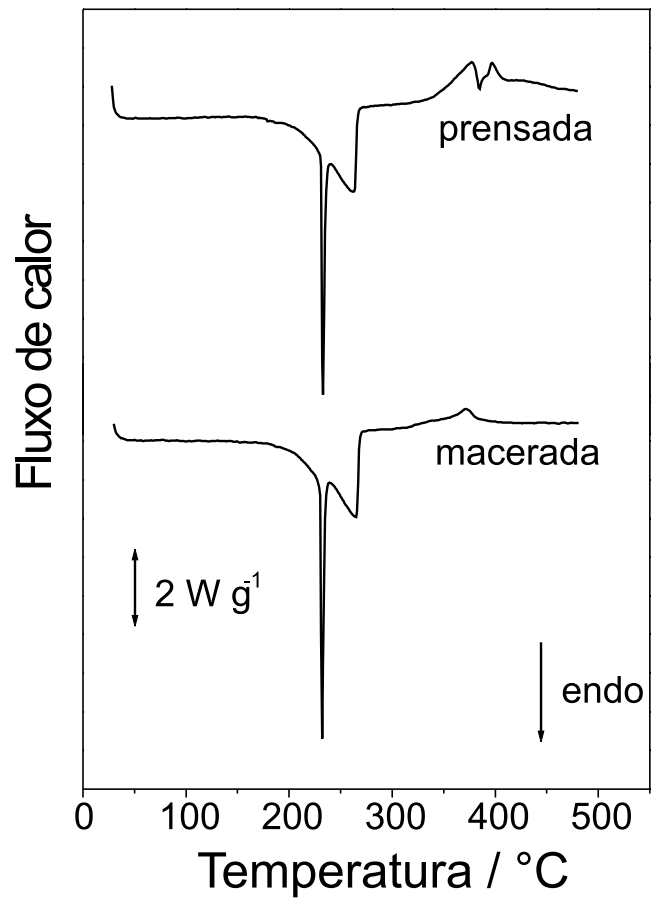

Figura 9. Curvas DSC da sacarina mostrando o efeito do adensamento da amostra. Massa de amostra $3 \mathrm{mg}$, suporte de amostra de alumínio com tampa, atmosfera dinâmica de ar sintético (100 $\left.\mathrm{mL} \mathrm{min}^{-1}\right)$, razão de aquecimento $10{ }^{\circ} \mathrm{C} \mathrm{min}^{-1}$

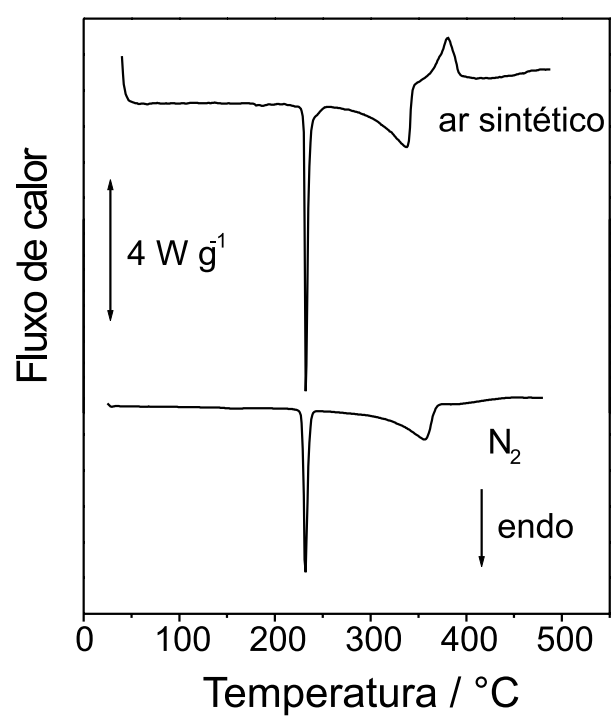

Figura 10. Curvas DSC da sacarina, com diferentes gases de arraste $\left(\mathrm{N}_{2} \mathrm{e}\right.$ ar, vazão $100 \mathrm{~mL} \mathrm{~min}^{-1}$ ), saturando a atmosfera do forno. Massa de amostra $3 \mathrm{mg}$, suporte de amostra de alumínio com tampa, razão de aquecimento $10{ }^{\circ} \mathrm{C} \mathrm{min}^{-1}$

um pico exotérmico em atmosfera de ar relativo à queima do material orgânico das impurezas e ausência deste em atmosfera de nitrogênio.

\section{CONCLUSÃO}

Pelos resultados apresentados, fica demonstrado que vários fatores são importantes para se obter resultados reprodutíveis em experi- 
mentos DSC. Assim, é fundamental relatar todos os parâmetros usados, como massa de amostra, natureza do suporte amostra, vazão e natureza do gás de arraste, razão de aquecimento, entre outros. Parâmetros referentes ao histórico da amostra também são importantes.

Alguns parâmetros podem ser manipulados pelo operador e outros, relacionados com as características fisico-químicas da amostra e da construção do equipamento não podem ser controlados, mas devem igualmente ser reportados para reprodutibilidade nos resultados.

É importante salientar que as técnicas diferenciais, tanto o DSC quanto o DTA, podem fornecer informações complementares ao TG, pois mesmo que não haja mudança na massa de amostra podem ocorrer eventos térmicos relacionados a eventos físicos, como fusão, sublimação, etc...

\section{REFERÊNCIAS}

1. Cavalheiro, E. T. G.; Ionashiro, M.; Breviglieri, S. T.; Marino, G.; Chierice, G. O.; Quim. Nova 1995, 18, 305.
2. Mackenzie, R. C.; Termochim. Acta 1979, 28,1 .

3. Ionashiro, M.; Giolito, I.; Cerâmica 1980, 26, 17

4. Wendlandt, W. W.; Thermal Analysis, $3^{\text {rd }}$ ed., Wiley: Nova Iorque, 1985, p. 213-460.

5. Hemminger, W. Em Calorimetry and Thermal Analysis of Polymers; Mathot, M.B.F., ed.; Hanser: Munique, 1994, p. 18-45.

6. Speil, S.; Berkelhamer, L. H.; Pask, J.A.; Davis, B.; U. S. Bur. Mines, Tech. Papers 1945, 664.

7. Kerr, P. F.; Kulp, J. L.; Am. Mineral. 1948, 33, 387.

8. Brown, M.E.; Introduction to Thermal Analysis, Chapman \& Hall: Nova Iorque, 1988.

9. Haines, P. J.; Thermal Methods of Analysis - Principles, Aplications and Problems, Champman \& Hall: Glasgou, 1995.

10. Gordon, S; J. Chem. Educ. 1963, 40, A87.

11. Willard, H.; Merrit Jr., L.; Dean, J.; Análise Instrumental, Tradução de Santos, M. S., 5 ${ }^{\text {a }}$ ed., Fundação Kalouste Goulbenkian: Lisboa, 1974, p. 579.

12. Skoog, D. A.; Leary, J. J.; Principles of Instrumental Analysis, $4^{\mathrm{a}}$ ed., Saunders: New York, 1992, p. 571

13. Tarbell, D. S.; Tarbell, A. T.; J. Chem. Educ. 1978, 55, 161.

14. Snyder, C. H.; The Extraordinary Chemistry of Ordinary Things, $2^{\mathrm{a}}$ ed., Wiley: New York, 1995, p. 546-548. 